\title{
Metabolism of a New Bactericidal Antiseptic, OPB-2045, in Rats following Subcutaneous Administration
}

\author{
Shoji Kudo, Ken Umehara, Masaaki Odomi and Gohachiro Miyamoto \\ Tokushima Research Institute, Otsuka Pharmaceutical Co., Ltd., Tokushima
}

\begin{abstract}
Summary: To investigate the metabolism and disposition of OPB-2045, 1-(3,4-dichlorobenzyl)-5-octylbiguanide, ${ }^{14} \mathrm{C}$-labeled OPB-2045 was subcutaneously injected to male Sprague-Dawley rats at a dose of 1 $\mathrm{mg} / \mathrm{kg}$ and the resulting radioactivity in the urine and bile was analyzed using a high-performance liquid radiochromatography. The radioactivity retained at the injection site was also analyzed.

1. The metabolites excreted in the urine included 6-[5-(3,4-dichlorobenzyl)-1-biguanidino $]$ hexanoic acid (DM-210) , 4-[5-(3,4-dichlorobenzyl)-1-biguanidino] butanoic acid (DM-212), 5-[5-(3,4-dichlorobenzyl) -1-biguanidino] pentanoic acid (DM-213) and 3,4-dichlorobenzoic acid. Within $72 \mathrm{hr}$ after the dosing, the metabolites DM-210, DM-212, DM-213, and 3,4-dichlorobenzoic acid excreted in the urine accounted for $4.6,1.6,1.5$ and $1.4 \%$ of the administered dose, respectively. No excretion of the unchanged compound was detected in the urine.

2. The metabolites excreted in the bile included DM-210, DM-212 and DM-213. The combined excretion rate of these metabolites accounted for $15.6 \%$ of the dose within $24 \mathrm{hr}$ postdosing. No excretion of 3,4dichlorobenzoic acid or the unchanged OPB-2045 was detected in the bile.

3. The radioactivity retained at the injection site at 1,8 and $24 \mathrm{hr}$ postdosing accounted for $51.5 \%$, $34.6 \%$ and $13.0 \%$ of the administered dose, respectively. The unchanged compound was found to account for at least $80.8 \%$ of the radioactivity, and no metabolites including DM-210, DM-212, DM-213 and 3,4dichlorobenzoic acid, were detected. These results indicated that OPB-2045 was not metabolized by the skin/tissues at the injection site, but instead by other organ(s).
\end{abstract}

Key words: OPB-2045, Metabolism, Subcutaneous administration, Rat

\section{Introduction}

OPB-2045 is a newly synthesized antimicrobial agent ${ }^{1)}$ and has potent bactericidal activity toward fungi (yeast), gram-negative and gram-positive bacteria including methicillin-resistant Staphylococcus aureus (MR$\mathrm{SA}$ ) and vancomycin-resistant Enterococci (VRE) at low concentrations. ${ }^{2)}$ The chemical structure of the compound is shown in Fig. 1.

The absorption, distribution and excretion of OPB2045 in rats and dogs following a single subcutaneous administration have been previously reported using ${ }^{14} \mathrm{C}$-labeled OPB-2045.,3) The disappearance of radioactivity from the circulation was relatively slow in either animal with half-lives of $58.6-63.3 \mathrm{hr}$ and the radioactivity retained at the injection site at $168 \mathrm{hr}$ postdosing accounted for $2 \%$ of the dose, showing slow elimination from the site.

In dogs, the urinary and fecal excretion of radioactivity within $168 \mathrm{hr}$ postdosing following the subcutaneous injection was $59.4 \%$ and $23.5 \%$ of the administered dose, respectively, demonstrating that most radioactivity was excreted in the urine. ${ }^{4)}$ Four OPB-2045 metabo- lites have been structurally identified using synthetic standards and/or spectral data as 6-[5-(3,4-dichlorobenzyl)-1-biguanidino] hexanoic acid (DM-210), 4[5-(3,4-dichlorobenzyl)-1-biguanidino] butanoic acid (DM-212), 5-[5-(3,4-dichlorobenzyl)-1-biguanidino] pentanoic acid (DM-213) and 3,4-dichlorobenzoic acid (Fig. 1).5) The predominant radioactive metabolites in the excreta were DM-212 and DM-213 in dogs.

The urinary and fecal excretion of radioactivity within $168 \mathrm{hr}$ postdosing was $17.2 \%$ and $68.4 \%$ of the dose, respectively, in rats given by the subcutaneous administration of ${ }^{14} \mathrm{C}-\mathrm{OPB}-2045$. However, no information on the metabolism of OPB-2045 in rats following subcutaneous administration is available. In the current study, the extent of the excretion of the unchanged OPB-2045 and metabolites in the urine and bile was studied in male Sprague-Dawley rats following a single subcutaneous administration of ${ }^{14} \mathrm{C}-\mathrm{OPB}-2045$ at a dose level of $1 \mathrm{mg} / \mathrm{kg}$. The radioactivity retained at the subcutaneous injection site was also analyzed using synthetic standards. 
<smiles>CCCCCCCCNC(=N)NC(=N)NC(C)c1ccc(Cl)c(Cl)c1</smiles><smiles>CC(C)OC(=O)CCCCCNC(=N)NC(=N)NCc1ccc(Cl)c(Cl)c1</smiles><smiles>CCNC(=N)NC(=N)NCc1ccc(Cl)c(Cl)c1</smiles><smiles>O=C(O)c1ccc(Cl)c(Cl)c1</smiles>

\section{3,4-dichlorobenzoic acid}

Fig. 1 Chemical structures of ${ }^{14} \mathrm{C}$-labeled $\mathrm{OPB}-2045$ and synthetic standards for OPB-2045 metabolites. Asterisk indicates the ${ }^{14} \mathrm{C}$-labeled position.

\section{Materials and Methods}

\section{Compound}

${ }^{14} \mathrm{C}$-labeled OPB-2045 monohydrochloride (lot No. C-921201) was synthesized at Nemoto \& Co., Ltd., (Tokyo, Japan). Specific radioactivity was $3.47 \mathrm{MBq} /$ $\mathrm{mg}$ and radiochemical purity, $99.3 \%$ as determined by a thin-layer chromatography using a silica gel $60 \mathrm{~F}_{254}$ plate in the solvent system, $\mathrm{CHCl}_{3}$ : methanol: $\mathrm{CH}_{3} \mathrm{COOH}$ (8: $3: 0.2$ ), or $98.4 \%$ determined by HPLC using a column of YMC A-302 (4.6 mm i.d. $\times 150 \mathrm{~mm}$; YMC Ltd., Corp., Kyoto, Japan) and mobile phase of methanol: water (85: 15) containing $2 \mathrm{~g} \mathrm{CH}_{3}\left(\mathrm{CH}_{2}\right)_{5} \mathrm{SO}_{3} \mathrm{Na}$ per one liter at a flow rate of $0.7 \mathrm{ml} / \mathrm{min}$. Non-radiolabeled OPB-2045 (lot No. 92K85M), DM-210 (lot No. 3K11M), DM-212 (lot No. 4H03M), and DM-213 (lot No. $4 \mathrm{H} 02 \mathrm{M}$ ) were provided by Otsuka Pharmaceutical Co., Ltd. 3,4-dichlorobenzoic acid was purchased from Wako Pure Chemical Industries Ltd., (Osaka, Japan). All other reagents used were of analytical grade.

\section{Experimental Animals}

Male Crj:CD ${ }^{\oplus}$ Sprague-Dawley rats at 6 weeks old were obtained from Charles River Japan Inc., and were acclimatized for more than one week in a controlled animal area maintained at 23 or $24 \pm 2^{\circ} \mathrm{C}$ for temperature, 55 or $60 \pm 10 \%$ for relative humidity and 13 times/ $\mathrm{hr}$ ventilation during 12-hr light/dark cycles. Rodent Chow MF (Oriental Yeast Co., Ltd., Japan) and water were available ad libitum under acclimatization. The animals at 7-8 weeks old (187-276 g) were used in the experiments.

\section{Dose Preparation and Administration}

${ }^{14} \mathrm{C}-\mathrm{OPB}-2045$ was dissolved in $5(\mathrm{w} / \mathrm{v}) \% \mathrm{D}$-mannitol (lot No. I21942; Kyowa Hakko Kogyo Co., Ltd.) aqueous solution for subcutaneous dosing at $3.47 \mathrm{MBq} /$ $1 \mathrm{mg} / \mathrm{kg} / 2.5 \mathrm{~m} l$ and injected into the dorsal part. The radiochemical purity of the dose preparation was more than $95.5 \%$ as judged by radio-HPLC.

\section{Sample Collection}

Following subcutaneous administration of ${ }^{14} \mathrm{C}-\mathrm{OPB}-$ 2045 , the rats were housed individually in metabolic cages and the excreted urine was collected at intervals of $24 \mathrm{hr}$ up to $72 \mathrm{hr}$ postdosing. The bile ducts of three rats were cannulated with polyethylene tubes for bile collection under ether anethesia and the animals were then subcutaneously administered with ${ }^{14} \mathrm{C}-\mathrm{OPB}-2045$ at 1 $\mathrm{mg} / \mathrm{kg}$, and placed in Bollman's cages. Bile was collected during $0-4,4-8$, and $8-24 \mathrm{hr}$ after dosing. Skin/tissues at the injection site were sampled at 1,8 , and $24 \mathrm{hr}$ postdosing.

\section{Analysis of Urinary and Biliary Radioactivity}

The urinary samples were centrifuged at $140 \times \mathrm{g}$ for 5 $\mathrm{min}$, and 5 to $20 \mathrm{~m} l$ of the supernatant was evaporated to dryness. The residue was suspended in $150 \mu l$ of methanol and centrifuged at $140 \times \mathrm{g}$ for $5 \mathrm{~min} ; 30 \mu \mathrm{l}$ of the resulting supernatant was analyzed by HPLC.

A total of $200 \mu l$ of bile was evaporated to dryness, and the residue was suspended in $150 \mu l$ of methanol. The suspension was centrifuged at $140 \times \mathrm{g}$ for $5 \mathrm{~min}$, and $30 \mu l$ of the resulting supernatant was analyzed by HPLC. 


\section{Analysis of Radioactivity at the Injection Site}

The skin/tissues at the injection site were cut into small pieces with scissors, mixed with $20 \mathrm{~m} l$ of methanol, and homogenized at $18000 \mathrm{rpm}$ for $5 \mathrm{~min}$ in a high-flex homogenizer (HF-92; Iuchi Corp., Japan). The homogenate was centrifuged at $140 \times \mathrm{g}$ for $5 \mathrm{~min}$, and the supernatant was obtained. This procedure was repeated three times and the extracts were combined. A portion $(0.5$ to $2 \mathrm{ml})$ of the extracted methanol fraction was dried, and the residue was then dissolved in $150 \mu l$ of methanol, and $30 \mu l$ of the resultant solution was analyzed by HPLC.

\section{High Performance Liquid Chromatography}

The following devices were used for HPLC: a Model 510 high-pressure pump (Waters), Model U6K universal injector (Waters), Model 484 tunable UV/VIS detector (Waters), Model 680 automatic gradient controller (Waters), and Model C-R7A chromatopak (Shimadzu).

A TSKgel ODS- $80_{\mathrm{TM}}$ column $(4.6 \mathrm{~mm}$ i.d. $\times 150 \mathrm{~mm}$; Tosoh, Tokyo, Japan) was used for the analysis of radioactivity in urine, bile and extracts of skin/tissues at the injection site. The unchanged OPB-2045 and metabolites were analyzed using a linear gradient developed from $10 \% \mathrm{CH}_{3} \mathrm{CN}$ in water containing $0.1 \%$ acetic acid to $80 \% \mathrm{CH}_{3} \mathrm{CN}$ in water containing $0.1 \%$ acetic acid over a period of $60 \mathrm{~min}$ at a flow rate of $1.0 \mathrm{ml} / \mathrm{min}$. The eluent was collected every $30 \mathrm{sec}$ into more than 120 fractions, and the radioactivity counted. The radioactivity peaks were identified by comparison with the elution time of the authentic standards, i.e.; unlabeled OPB-2045, DM-210, DM-212, DM-213, and 3,4dichlorobenzoic acid.

\section{Radiochemical Analysis}

The samples were mixed with $0.5-1.0 \mathrm{~m} l$ water and 8 $\mathrm{m} l$ ACS-II scintillant solution (Amersham, UK), and radioactivity was counted for one min with a liquid scintillation counter (LSC-1050 and -3500; Aloka, Tokyo, Japan). The counting efficiency was assessed by an external standard method.

\section{Results}

\section{Radioactivity in Urine}

Fig. 2 shows a representative HPLC radiogram of the urine, and Table I shows the urinary excretion rates of the unchanged OPB-2045 and metabolites at $72 \mathrm{hr}$ after subcutaneous injection of ${ }^{14} \mathrm{C}-\mathrm{OPB}-2045$ at a dose of 1 $\mathrm{mg} / \mathrm{kg}$.

In the radiochromatogram, several radioactive metabolites including DM-210, DM-212, DM-213 and 3,4-dichlorobenzoic acid appeared in the urine. The unidentified radioactive metabolites were also detected in the urine, however, the unchanged OPB-2045 did not appear in any samples.

Within $72 \mathrm{hr}$ of the subcutaneous administration of ${ }^{14} \mathrm{C}-\mathrm{OPB}-2045$ at $1 \mathrm{mg} / \mathrm{kg}, 14.2 \%$ of the administered

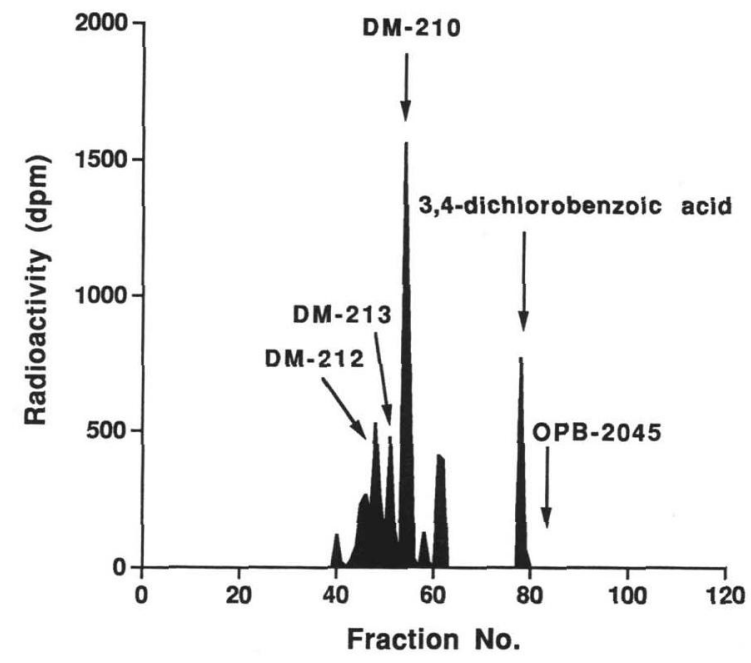

Fig. 2 Representative radio-HPLC chromatogram of a typical urine $(0-24 \mathrm{hr})$ extract from a rat containing OPB2045 metabolites.

Table I Urinary excretion of OPB-2045 and its metabolites in rats following a single subcutaneous administration of ${ }^{14} \mathrm{C}-\mathrm{OPB}-2045$ at $1 \mathrm{mg} / \mathrm{kg}$

\begin{tabular}{lc}
\hline OPB-2045 or metabolite & $\begin{array}{c}\text { Excretion rate } \\
(\% \text { of dose })\end{array}$ \\
\hline OPB-2045 & n.d. \\
DM-210 & $4.6 \pm 0.6(32.4)$ \\
DM-212 & $1.6 \pm 0.1(11.3)$ \\
DM-213 & $1.5 \pm 0.3(10.6)$ \\
3,4 -dichlorobenzoic acid & $1.4 \pm 0.8(9.9)$ \\
Others & $5.0 \pm 0.6(35.2)$ \\
\hline Total & $14.2 \pm 1.0(100)$ \\
\hline
\end{tabular}

The urinary sample was collected up to $72 \mathrm{hr}$ postdosing. Values represent mean \pm SD of three animals.

Values in parentheses are expressed as the percent content of each substance to the total excreta of radioactivity in urine. n.d., not detected.

dose of radioactivity was excreted in the urine. The rate of DM-210 excretion was particularly high (4.6\%) in relation to the administered dose and accounted for $32.4 \%$ of the urinary radioactivity. The metabolites DM-212, DM-213, and 3,4-dichlorobenzoic acid excreted in the urine accounted for $1.6 \%, 1.5 \%$, and $1.4 \%$, respectively, of the administered dose.

\section{Radioactivity in Bile}

Fig. 3 shows a representative HPLC radiogram of the bile, and Table II shows biliary excretion rates of radioactive substances.

Within $24 \mathrm{hr}$ of the subcutaneous administration of ${ }^{14} \mathrm{C}-\mathrm{OPB}-2045,34.1 \%$ of the administered dose of radioactivity was excreted in the bile. The metabolites excreted in the bile included DM-210, DM-212, and DM-213. The combined excretion rate of these metabolites accounted for $15.6 \%$ of the administered dose and 


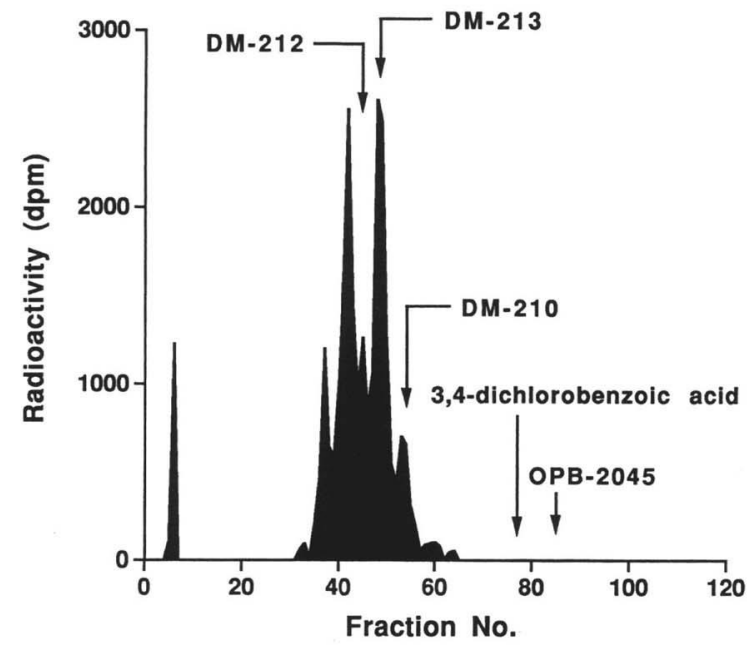

Fig. 3 Representative radio-HPLC chromatogram of a typical bile (4-8 hr) extract from a rat containing OPB2045 metabolites.

Table II Biliary excretion of OPB-2045 and its metabolites in rats following a single subcutaneous administration of ${ }^{14} \mathrm{C}-\mathrm{OPB}-2045$ at $1 \mathrm{mg} / \mathrm{kg}$

\begin{tabular}{lc}
\hline OPB-2045 or metabolite & $\begin{array}{c}\text { Excretion rate } \\
(\% \text { of dose })\end{array}$ \\
\hline OPB-2045 & n.d. \\
DM-210+DM-212+DM-213 & $15.6 \pm 3.3(45.7)$ \\
3, 4-dichlorobenzoic acid & n.d. \\
Others & $18.5 \pm 5.7(54.3)$ \\
\hline Total & $34.1 \pm 9.0(100)$ \\
\hline
\end{tabular}

The biliary sample was collected up to $24 \mathrm{hr}$ postdosing. Values represent mean \pm SD of three animals.

Values in parentheses are expressed as the percent content of each substance to the total excreta of radioactivity in bile. n.d., not detected

$45.7 \%$ of the biliary radioactivity. No excretion of $3,4-$ dichlorobenzoic acid or the unchanged OPB-2045 was detected.

\section{Radioactivity at the Injection Site}

The radiochromatograms of the extracts from the injection site are shown in Fig. 4.

The radioactivity retained at the injection site at 1,8 and $24 \mathrm{hr}$ postdosing accounted for $51.5 \%, 34.6 \%$ and $13.0 \%$ of the administered dose, respectively. The unchanged compound was found to account for at least $80.8 \%$ of the radioactivity, and no metabolites including DM-210, DM-212, DM-213 and 3,4-dichlorobenzoic acid were detected.

\section{Discussion}

In our previous findings, the urinary and fecal excretion of radioactivity following the subcutaneous administration of ${ }^{14} \mathrm{C}-\mathrm{OPB}-2045$ was $17.2 \%$ and $68.4 \%$, respectively, in rats and $59.4 \%$ and $23.5 \%$, respectively, in
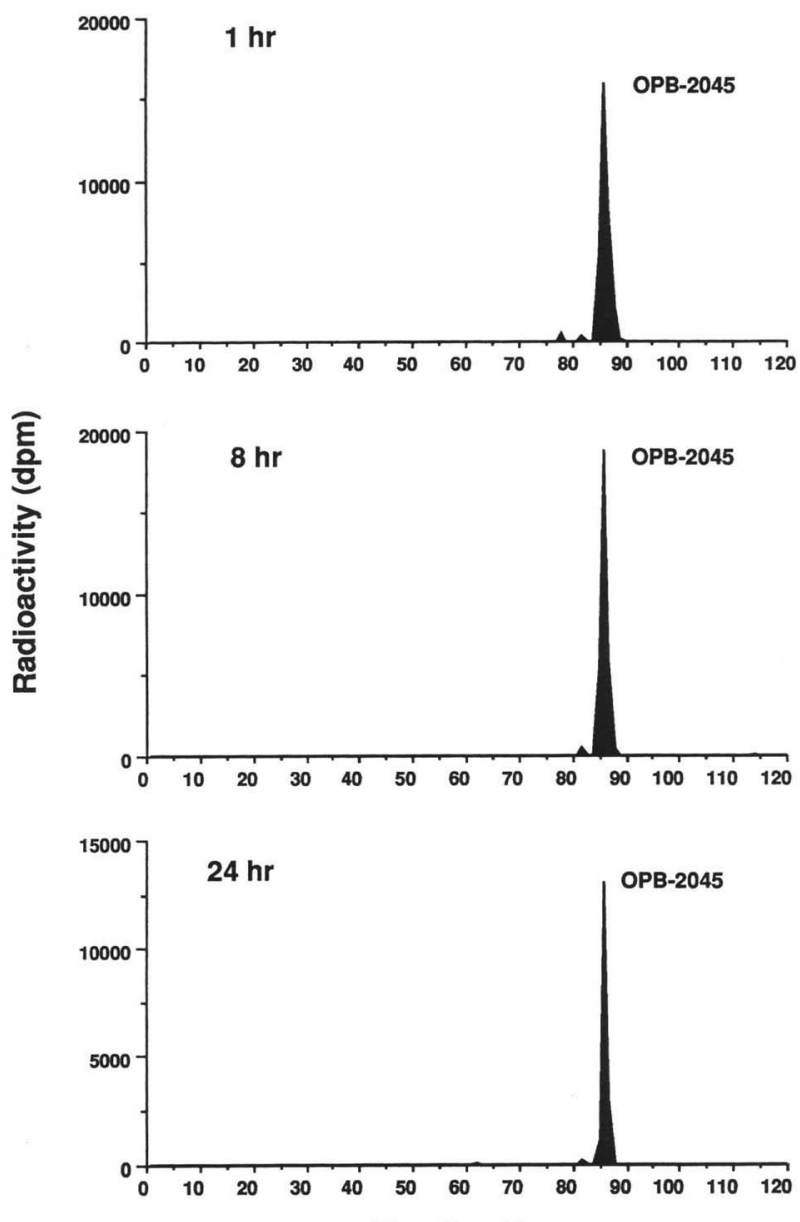

Fraction No.

Fig. 4 Representative radio-HPLC chromatograms of a typical extract from the injection site following a single subcutaneous injection of ${ }^{14} \mathrm{C}-\mathrm{OPB}-2045$ at $1 \mathrm{mg} / \mathrm{kg}$ to rats.

dogs within $168 \mathrm{hr}$ postdosing, showing species differences due to the different route of excretion of the radioactivity. ${ }^{3,4)}$ The metabolic fate of OPB-2045 in dogs following subcutaneous administration has been previously reported that OPB-2045 was extensively biotransformed into various metabolites and the excretion of the unchanged OPB-2045 was quite low in the feces, and was not observed in the urine. ${ }^{5)}$ Four metabolites of OPB-2045 have been structurally identified from the dog urine, i.e.; DM-210, DM-212, DM-213 and 3,4dichlorobenzoic acid. ${ }^{5)}$ With this background, this study was conducted to investigate whether the metabolic fate of OPB-2045 in rats and dogs was similar or not.

Following the subcutaneous administration of OPB2045 to rats, it was extensively biotransformed into the various metabolites, and no urinary and biliary excretions of the unchanged OPB-2045 was observed. This demonstrated that OPB-2045 was eliminated from the body due to the metabolic clearance, as was the case in dogs.

The excreted metabolites in the rats included $\mathrm{DM}_{-}^{-}$ 
210, DM-212, DM-213 and 3,4-dichlorobenzoic acid in the urine, and the same metabolites except 3,4dichlorobenzoic acid were found in the bile. In the dogs, the same four metabolites were detected in the urine, and the excretion of these metabolites except 3,4dichlorobenzoic acid was observed in the feces. ${ }^{5}$ ) This evidence indicated that the metabolic profile of OPB2045 in the rats following subcutaneous administration was similar to that in the dogs, and additionally $3,4^{-}$ dichlorobenzoic acid was probably not excreted in the bile.

The predominant metabolite in the urinary excreta was DM-210 in the rats, accounting for $32.4 \%$ of the urinary radioactivity, but it was DM- 213 followed by DM210 in dogs, accounting for $33.3 \%$ and $31.8 \%$ of the radioactivity in the urine, respectively.5) $\mathrm{DM}-210$ was thus one of the major metabolites of OPB-2045 in either animals.

The biotransformation of OPB-2045 to 3,4-dichlorobenzoic acid is considered to occur by several metabolic processes, and the enzymes involved in the process are considered to be a cytochrome P450 system and/or flavin monooxygenase, and aldehyde dehydrogenase. ${ }^{5)}$ On the other hand, the octyl group of OPB-2045 was predominantly metabolized and biotransformed to butanoic (DM-212), pentanoic (DM-213) and hexanoic acid (DM-210) in rats as well as in dogs. These metabolites were all a methylene chain shorter with a carboxyl end and thus produced by $\mathrm{C}-\mathrm{C}$ bond cleavage followed by subtraction of carbon fragments. The mechanism (s) of production of these $\mathrm{C}-\mathrm{C}$ bond cleavage metabolites was still unclear, but, it was clear in this study that rats could metabolize OPB-2045 to these degraded products as was the case in the dogs. Investigation regarding the mechanism(s) of the $\mathrm{C}-\mathrm{C}$ bond cleavage of the octyl group is now in progress.

The elimination of the radioactivity retained at the injection site was relatively slow, however, the radioactivi- ty decreased time-dependently. Radioactivity at the injection site originated from the unchanged OPB-2045 at any time postdosing and no metabolites including DM210, DM-212, DM-213 and 3,4-dichlorobenzoic acid were detected. This evidence indicated that OPB-2045 was not metabolized by the skin/tissues at the injection site, but instead by the other organ (s).

In conclusion, OPB-2045 following subcutaneous administration was eliminated from the body by the metabolic clearance in rats and the metabolic profile of the compound in the animal was fundamentally similar to that in dogs. Furthermore, it suggested that species difference between these animals due to the different route of excretion of the radioactivity following subcutaneous administration was probably based on the excretory ability of each metabolite into urine or bile.

\section{References}

1) Tsubouchi, H., Ohguro, K., Yasumura, K., Ishikawa, H., and Kikuchi, M.: Synthesis and structure-activity relationships of novel antiseptics. Bioorg. Med. Chem. (Lett.) 7: 1721-1724 (1997).

2) Ohguro, K., Ohnishi, H., Tomigashi, N., Tsubouchi, H., Yasumura, K., and Kikuchi, M.: Bactericidal activity of OPB-2045: A new antiseptic. 20 International Congress of Chemotherapy, Sydney, Australia, pp. 51 (1997).

3) Kudo, S., Iwasaaki, M., Sugimoto, K., Kodama, R., and Odomi, M.: Absorption, distribution and excretion of OPB2045 following a single subcutaneous administration to rats. Xenobio. Metabol. Dispos., 13: 1-7 (1998).

4) Kudo, S., Iwasaaki, M., Sugimoto, K., Kodama, R., and Odomi, M.: Absorption, distribution and excretion of OPB2045 following a single subcutaneous administration to beagle dogs. Xenobio. Metabol. Dispos., 13: 8-12 (1998).

5) Kudo, S., Umehara, K., Morita, S., Uchida, M., Miyamoto, G., and Odomi, M.: Metabolism of 1-(3,4-dichlorobenzyl)5-octylbiguanide in the dog. Xenobiotica, 28(5): 507-514 (1998). 